\title{
Cetáceos de las aguas costeras del Pacífico norte y sur de Costa Rica
}

\author{
Damián Martínez-Fernández ${ }^{1,2}$, Andrea Montero-Cordero $^{1} \&$ Laura May-Collado $^{3}$ \\ 1. Fundación Keto, Apdo. 1735-1002 San José, Costa Rica; dmartinez@ fundacionketo.org \\ 2. Instituto Internacional en Conservación y Manejo de Vida Silvestre, Universidad Nacional. Apdo 1350-3000 Heredia \\ Costa Rica; amontero@fundacionketo.org \\ 3. Departamento de Biología Universidad de Puerto Rico, Rio Piedras Puerto Rico; 1maycollado@gmail.com
}

$$
\text { Recibido 04-V-2010. Corregido 20-IX-2010. } \quad \text { Aceptado 19-X-2010. }
$$

\begin{abstract}
Inshore cetaceans from the North and South Pacific coast of Costa Rica. Twenty nine cetacean species occur in Costa Rican waters but extensive research has been conducted only for three species. The latter shows there is a lack of general and local information about these mammals, even when the country, has shown a remarkable growth in whale watching activities. The increasing use of marine resources in coastal areas has also developed the need to determine the occurrence of cetaceans in areas showing high tourist presence, in order to propose sound conservation measures. In this study, environmental variables were determined and subsequently related to the presence of the species recorded, out of 166 sightings, between 2005 and 2006. The species with highest proportion of sightings were Stenella attenuata (68\%), followed by Megaptera novaeangliae (13\%) and Tursiops truncatus (10\%). The presence of spotted dolphins is related to changes in salinity and water transparency, while that of the humpback whale was related to wave height (Beaufort scale) and water temperature. The presence of seven species of cetaceans was confirmed in two coastal areas of the Pacific coast of Costa Rica, from which three are present throughout the year. Environmental variables were found related to the presence of at least two species. Rev. Biol. Trop. 59 (1): 283-290. Epub 2011 March 01.
\end{abstract}

Key words: whales, dolphins, Costa Rica, Stenella, Tursiops, Megaptera, cetaceans, Pacific coast.

A finales de los años ochenta, la diversidad de los mamíferos marinos de Costa Rica registraba únicamente siete especies de cetáceos: el delfín manchado (Stenella attenuata), el delfín rotador (Stenella longirostris), el delfín común (Delphinus delphis), el calderón menor (Peponocephala electra), la orca (Orcinus orca), el calderón gris (Grampus griseus), el cachalote (Physeter macropcephalus) y el manatí (Trichechus manatus) (Janzen \& Wilson 1983). Luego en 1996 un estudio regional para la zona reportó las primeras estimaciones de abundancia de 12 especies de cetáceos en la Zona Económica Exclusiva (ZEE) del Pacífico de Costa Rica (Gerrodette \& Palacios 1996). La revisión más reciente realizada para el país estima un total de 30 especies de cetáceos (MayCollado 2009). Sin embargo, de éstas sólo 19 han sido confirmadas en aguas oceánicas del Pacífico (May-Collado et al. 2005).

Hoy día se reconoce para al país un total de 34 especies de mamíferos marinos, incluyendo 30 cetáceos, un sirénido, y tres pinnipedios, representando cerca del $26 \%$ de la diversidad mundial de mamíferos marinos (May-Collado 2009). Aun así los estudios en aguas costeras del Pacífico nacional se han concentrado en tres especies: el delfín nariz de botella o bufeo (Tursiops truncatus) (Acevedo-Gutiérrez \& Burkhart 1998, Cubero-Pardo 1998, CuberoPardo 2007, Oviedo 2007, Acevedo-Gutiérrez 1999, Acevedo-Gutiérrez \& Parker 2000, Acevedo-Gutiérrez \& Stienessen 2004, PalaciosAlfaro 2007), el delfín manchado (Stenella attenuata) (Acevedo-Gutiérrez \& Burkhart 1998, Cubero-Pardo 1998, May-Collado \& 
Forcada 2001, May-Collado \& Morales-Ramírez 2005, Montero-Cordero 2007, MonteroCordero \& Lobo 2010) y la ballena jorobada (Megaptera novaeangliae) (Steiger et al. 1991, Acevedo-Gutiérrez \& Smultea 1995, Calambokidis et al. 2000, Rassmussen et al. 2007, Oviedo \& Solís 2008).

A pesar de toda esta diversidad y los estudios que se han realizado, la información que se tiene es poca para poder generar estrategias de conservación de los cetáceos costeros del país. Actualmente la mitad de las especies de cetáceos encontradas en aguas costarricenses están bajo la categoría de "datos deficientes" según la Lista Roja de la UICN. Paradójicamente, el avistamiento de cetáceos en Costa Rica representa la economía con mayor crecimiento de América (Hoyt \& Iniguez 2008). Es por esto que es necesario determinar la presencia de cetáceos costeros en zonas de actividad turística del Pacifico del país y estimar la influencia de algunos parámetros ambientales para su ocurrencia como se ha reportado en otras zonas costarricenses. (Acevedo-Gutiérrez \& Burkhart 1998, May-Collado \& Forcada 2001, May-Collado \& Morales-Ramírez 2005, Cubero-Pardo 2007, Oviedo 2007).

\section{MATERIALES Y MÉTODOS}

Área de estudio: El estudio se realizó en dos zonas, 1) Isla del Caño (IC): se ubica en el Pacífico sur de Costa Rica y el área se delimitó mediante cuatro triángulos imaginarios de $60 \mathrm{~km}$ de perímetro que se distribuyeron al norte, sur, este y oeste de la Isla del Caño. La zona se caracteriza por tener un clima lluvioso (abril-diciembre) y una época seca poco pronunciada (Quesada et al. 2006). Durante las lluvias se incrementa el nivel de sedimentos y nutrimentos en el sistema marino y durante la estación seca se registran las máximas temperaturas atmosféricas (Quesada-Alpízar \& Cortés 2006) y 2) Bahía Santa Elena (BS): se ubica en el Pacífico norte y el área de trabajo se delimitó mediante un triángulo imaginario de $60 \mathrm{~km}$ de perímetro desde Isla Bolaños dentro de Bahía Salinas hasta las islas Murciélago en el Parque Nacional Santa Rosa y de ahí hasta la comunidad de Cuajiniquil. Esta zona presenta la dinámica de las estaciones generales del país y sigue el patrón presentado por Waylen \& Caviedes (1996), donde existe una época de lluvias entre mayo-noviembre y una época seca entre diciembre-abril muy pronunciada. Esta zona contiene las aguas más productivas de la región norte de Costa Rica (Brenes 2001).

Registro de datos: Basados en estudios previos en Isla del Caño (Montero-Cordero 2007) e Isla Murciélago (e.g. May-Collado \& Morales-Ramírez 2005), se realizaron recorridos sistemáticos en bote fuera de borda con dos personas. Entre mayo 2005 y abril 2006 en los dos perímetros de las áreas de estudio se siguieron transectos de banda de un $\mathrm{km}$ de ancho para ubicar cetáceos.

Con base en trabajos previos con cetáceos costeros (May-Collado et al. 2005 y MonteroCordero 2007) se adaptó una metodología de tres o cuatro días consecutivos de muestreos mensuales, con un promedio de ocho horas de esfuerzo de muestreo entre las 06:00 y 14:00hr de cada día.

En Costa Rica se ha reportado la influencia indirecta de factores ambientales en la presencia de cetáceos (May-Collado \& Forcada 2001, May-Collado \& Morales-Ramírez 2005, Cubero-Pardo 2007, Oviedo 2007). Durante los recorridos se tomaron datos de temperatura superficial in situ y satelital $\left({ }^{\circ} \mathrm{C}\right)$, salinidad (\%o), oleaje (Beaufort), nubosidad (1/8), concentración de clorofila $\alpha$ y transparencia del agua (m). La toma de estos datos se realizó en los siguientes periodos del día: 0600-0900, 0900-1200 y 1200-1500hr. Para el análisis de los datos promedio diarios de temperatura superficial satelital y concentración de clorofila $\alpha$ se utilizaron imágenes satelitales pertenecientes al proyecto Giovanni de la NASA, basados en Ocean Biology Processing Group (OBPG), con formato SMI (Standard Mapped Image) con una resolución espacial MODIS/ AQUA de 9 kilómetros. Se utilizaron estas imágenes satelitales ya que eran de acceso 
libre, digitales, procesadas en formato png y listas para la interpretación.

Para determinar la asociación entre la riqueza de especies por época seca o de lluvia y el número de animales observados y los tamaños grupales (rangos entre 0-10, 10-20, $30-40$, hasta 500), por zona de trabajo se realizó con un análisis $\chi^{2}$ de asociación con el programa Infostat 1.1 (InfoStat 2003). Para predecir las especies de cetáceos con mayor frecuencia de observación, se utilizó un modelo de regresión logística (GLM). Se analizó la presencia o ausencia de especies y su relación con una o más variables ambientales mediante una distribución binomial y función logit, según los Criterios de Información de Akaike (AIC) (Akaike 1974) con en el programa R v.2.3.0 (R Development Core Team 2006). Este análisis se realizó para las especies que presentaron mayor frecuencia de avistamiento durante el año.

\section{RESULTADOS}

A pesar de las diferencias en clima entre IC y BS se pudo invertir un total de $431 \mathrm{hr}$ efectivas de muestreo en 56 días $\left(\mathrm{IC}_{\text {horas }}=272\right.$, $\mathrm{IC}_{\text {días }}=34, \mathrm{BS}_{\text {horas }}=159, \mathrm{BS}_{\text {días }}=22$ ) entre los meses de mayo 2005 a abril 2006. Los recorridos mensuales se realizaron en un área aproximada de $700 \mathrm{~km}^{2}$ en IC y $174 \mathrm{~km}^{2}$ en BS. La distribución de los avistamientos varió a lo largo del año en IC y BS (Fig. 1). Se registraron 7 especies en un total de 166 avistamientos y un promedio general de avistamientos por kilómetro recorrido de $0.042( \pm 0.025)$ en IC y de $0.073( \pm 0.079)$ en BS (Cuadro 1).

De todas las especies solo se obtuvieron datos suficientes para la comparación de tres de ellas: S. attenuata, T. truncatus y M. novaeangliae. La única que presentó un número de avistamientos mayor por área de estudio fue el delfín manchado $\left(\mathrm{ICn}=89, \mathrm{BSn}=22, \chi^{2}=20.91\right.$,
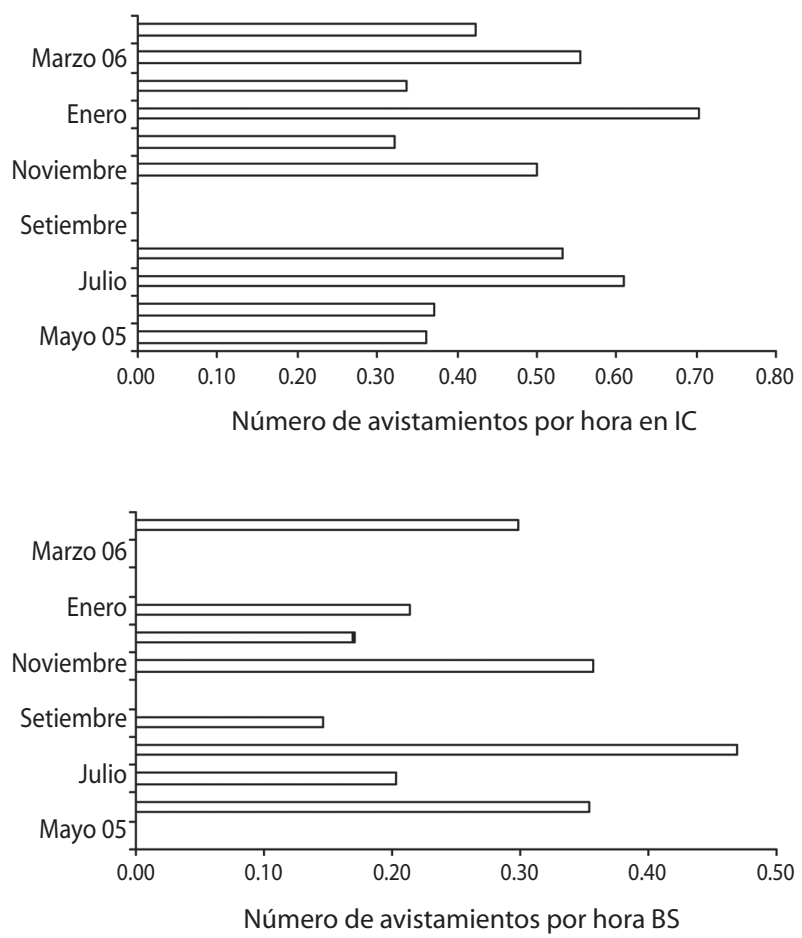

Fig. 1. Distribución de avistamientos por hora a lo largo del año en IC y BS.

Fig. 1. Sighting distribution (sightings per hour) throughout the year in IC and BS. 
CUADRO 1

Número de avistamientos $(N)$, número total de animales observados por kilómetro recorrido $(A)$ e Índice de avistamientos por kilómetro recorrido $(\mathrm{Av} / \mathrm{km})$

TABLE 1

Sighting number $(N)$, total of sighted animals per $\mathrm{km}(\mathrm{A})$ and sighting index (Av/km)

\begin{tabular}{|c|c|c|c|c|c|c|c|c|c|}
\hline \multirow{2}{*}{ Especie } & \multirow{2}{*}{ Nombre común } & \multicolumn{4}{|c|}{ BS } & \multicolumn{4}{|c|}{ IC } \\
\hline & & $\mathrm{N}$ & $\%$ & A & $\mathrm{Av} / \mathrm{km}$ & $\mathrm{N}$ & $\%$ & A & $\mathrm{Av} / \mathrm{km}$ \\
\hline S. atenuatta & Manchado & 23 & $55 \%$ & 0.504 & 0.024 & 89 & $69 \%$ & 0.885 & 0.025 \\
\hline M. novaeangliae & Ballena jorobada & 10 & $29 \%$ & 0.0204 & 0.010 & 12 & $10 \%$ & 0.005 & 0.003 \\
\hline T. truncatus & Nariz de botella & 3 & $7 \%$ & 0.150 & 0.003 & 14 & $10 \%$ & 0.041 & 0.004 \\
\hline P. crassidens & Falsa orca & 0 & $0 \%$ & 0 & 0 & 7 & $6 \%$ & 0.016 & 0.002 \\
\hline O. orca & Orca & 3 & $2 \%$ & 0.007 & 0.003 & 0 & $0 \%$ & 0 & 0 \\
\hline S. bredanensis & Dientes rugosos & 0 & $0 \%$ & 0 & 0 & 2 & $2 \%$ & 0.016 & 0.0006 \\
\hline D. delphis & Común & 0 & $0 \%$ & 0 & 0 & 1 & $1 \%$ & 0.112 & 0.0003 \\
\hline
\end{tabular}

$\mathrm{gl}=09, \mathrm{p}=0.01)$. Además hubo una tendencia a la presencia estacional de la ballena jorobada en ambas áreas, donde aumentaron los avistamientos en la época de lluvias en IC y a inicios de época seca en BS, sin embargo; los avistamientos en ambas zonas fueron consistentes a lo largo del año $\left(\chi^{2}=9.47, \mathrm{gl}=05, \mathrm{p}=0.09\right)$.

Los tamaños grupales en BS y IC variaron por especie. Para los delfines manchados prevalecieron los grupos con menos de 10 animales $\left(\chi^{2}=34.59, \mathrm{gl}=18, \mathrm{p}=0.01\right)$. Para el delfín nariz de botella predominaron grupos más grandes (> 20 individuos) en ambas zonas $\left(\chi^{2}=165.14, \mathrm{gl}=50, \mathrm{p}<0.01\right)$. Los grupos de ballenas jorobadas no sobrepasaron los dos individuos. El delfín común, el delfín de dientes rugosos y la falsa orca se observaron en grupos grandes $(>100)$, pero de manera esporádica ( $<$ de 3 avistamientos en este estudio).

De las especies que se avistaron con mayor frecuencia a lo largo del año (únicamente $S$. attenuata y $M$. novaenagliae) se pudieron determinar los factores ambientales que pueden afectar la presencia indirecta. Para los delfines manchados fueron la salinidad y transparencia del agua en la zona de IC. Para la ballena jorobada la temperatura resultó la variable del modelo en BS y el oleaje en IC. En el Cuadro 2 , se muestran estos factores ambientales para cada especie por zona basado en un modelo de regresión logística.

\section{DISCUSIÓN}

Las tres especies de cetáceos más observados a nivel costero en los alrededores de Bahía Santa Elena e Isla del Caño en este estudio fueron S. attenuata, T. truncatus y M. novaeangliae. Los resultados concuerdan con lo reportado por May-Collado et al. (2005). Dichos autores utilizaron una base de datos más amplia pero en su mayoría oceánica (1979-2001) e informaron que las especies más avistadas en aguas costeras del Pacífico nacional son las ballenas jorobadas, los delfines manchados y nariz de botella, mientras que en aguas oceánicas es el delfín listado. Esto podría explicar que éstas hayan sido las especies más estudiadas en el país en los últimos 15 años, a pesar de la alta riqueza presente en las aguas patrimoniales. Aún cuando su observación fue ocasional para este estudio, la presencia de la falsa orca, orca o el delfín de dientes rugosos, parecen ser de relevancia para la riqueza en las costa Pacífica costarricenses como ha sido informado en otros trabajos (Fertl et al. 1996, Acevedo-Gutiérrez et al. 1997, Martínez-Fernández et al. 2005, Martínez-Fernández \& Garita-Alpízar 2006, May-Collado et al. 2005).

Los avistamientos fueron dominados por grupos del delfín manchado, especie siempre presente a través del año de muestreo con un alto número de individuos durante el inicio de 


\section{CUADRO 2}

Selección de modelos de regresión logística ${ }^{a}$ sobre la presencia de delfines manchados y ballenas jorobadas para una o más variables ambientales ${ }^{b}$ en la zona del Pacífico norte y sur de Costa Rica

TABLE 2

Model selection of logistic regression a for the presence of spotted dolphins and humpback whales for one or more variables ${ }^{b}$ in the North and South coast of Costa Rica

$\begin{array}{cccccccc}\text { Zona } & \text { Modelo } & \mathrm{k} & \mathrm{w} & \mathrm{AICc}^{\mathrm{a}} & \text { Variable }^{\mathrm{b}} & \text { Coeficiente } & \text { IC 95\% } \\ \text { IC } & \text { Sag (sal + secc) } & 3 & 0.38 & 33.594 & \text { Sal } & 2.00 & 0.96,4.80 \\ & & & & & \text { Secc } & 0.83 & 0.69,0.96 \\ \text { BS } & \text { Sag (nulo) } & 1 & 0.28 & 29.142 & \text { Nulo } & 1.50 & 0.62,3.82 \\ \text { IC } & \text { Mn (ola) } & 2 & 0.41 & 35.313 & \text { Ola } & 0.105 & 0.01,0.58 \\ \text { BS } & \text { Mn (ti) } & 2 & 0.32 & 29.575 & \text { Ti } & 0.720 & 0.45,1.06\end{array}$

a: los resultados incluyen Criterios de Información de Akaike corregidos por pequeño tamaño de muestra (AICc), AICc relativo ( $\triangle \mathrm{AICc}$ ), peso de Akaike (w) y número de parámetros $(\mathrm{k})$.

b: ti=temperatura superficial in situ, Cloro=concentración de clorofila $\alpha$, Ts=temperatura superficial satelital, ola=oleaje, sal=salinidad, secc=transparencia.

a: Results include Akaike's Information Criterion corrected for small sample size (cAIC), relative cAIC ( $\triangle$ AICc), Akaike weight (w) and number of parameters (k).

b: Ti=in situ surface temperature, Cloro $=\alpha$ chlorophyll concentration, Ts=satellite surface temperature, Ola=beaufort, Sal=salinity, Secc=transparency.

los meses de lluvias (Mayo - Junio). El dominio de los avistamientos era de esperar, ya que el delfín manchado es de las especies más abundantes en el Pacífico Tropical Oriental (Perrin 2002) y se ha sugerido la existencia de una población costera residente para Costa Rica y Panamá (Escorza-Trevino et al. 2005). Para la ballena jorobada se confirman los patrones de ocurrencia estacionales establecidos anteriormente (Clapham \& Mead 1999, Flórez-González et al. 1998). Estas migran anualmente de sus sitios de nacimiento en aguas tropicales (e.g. Costa Rica) a los sitios de alimentación en zonas templadas (Calambokidis et al. 2000, Rasmussen et al. 2007).

La ballena jorobada esta presente en IC y BS durante el año, debido a que individuos de ambas poblaciones aparecen en aguas costarricenses durante las épocas de reproducción (Acevedo-Gutiérrez \& Smultea 1995).

Los tamaños de grupos encontrados a lo largo de la costa para las tres especies más comunes fueron pequeños, menores a 20 individuos. En el caso del delfín manchado y nariz de botella, los grupos pequeños coinciden con lo reportado anteriormente (May-Collado \& Morales-Ramírez 2005, García \& Dawson 2003, Montero-Cordero 2007, Palacios 2007) y podrían reflejar el efecto de la disminución de sus depredadores en aguas costeras (Norris \& Dohl 1980, Connor et al. 2000). En el caso de la ballena jorobada, a la fecha en las zonas de reproducción de Costa Rica se esperan grupos de machos $>2$ individuos, individuos solitarios, parejas madre-cría. Este último ensamble fue el dominante en los avistamientos de IC y BS para este estudio a diferencia de lo reportado en otros estudios (Rassmussen et al. 2007, Oviedo \& Solís 2008).

La presencia del delfín manchado se vio influenciada por variables ambientales (salinidad y transparencia de agua) que resultaron dependientes de la época seca en IC. Aunque la cantidad animales aumentó a inicios de la época lluviosa, el mayor número de avistamientos se obtuvo durante los meses secos, los cuales poseen factores ambientales que favorecen su presencia. Este aumento de avistamientos durante esta época seca puede deberse a que hubo agregaciones de alimentación en áreas 
cercanas a la Isla del Caño, lo cual favoreció su observación. En BS la inconstancia de las variables ambientales medidas no permitió hacer una predicción para delfines manchados y el modelo resultó nulo. Para ballenas jorobadas en IC la disminución del oleaje en el verano aumenta la probabilidad de observación, por lo que la facilidad del avistamiento puede afectar los datos. En BS la temperatura baja coincide con el patrón de migración de ballenas, en donde se pueden observar más individuos de la población del norte entre diciembre y marzo. Para esta época un afloramiento es el responsable de los cambios estacionales en la temperatura $\left(<22^{\circ} \mathrm{C}\right)$ (Brenes et al. 1995 , Jiménez 2001).

En conclusión, se confirma la presencia de 7 especies de cetáceos en dos zonas costeras del Pacífico de Costa Rica y tres se presentan a lo largo del año. Además, se encontraron variables ambientales que se relacionaron con la presencia de al menos 2 especies. Toda esta información favorece el entendimiento de las especies costeras para sectores en donde los cetáceos se han vuelto un recurso valioso, como es el caso del turismo (Montero-Cordero 2007, Montero-Cordero \& Martínez 2007, Rodríguez-Fonseca \& Fischel-Quirós 2007). Por ser especies carismáticas, la valoración de delfines y ballenas presentes en la costa favorecen la ampliación o consolidación de áreas marinas protegidas, como por ejemplo para el Parque Nacional Santa Rosa, el Parque Nacional Mario Ballena y la Reserva Biológica Isla del Caño.

\section{AGRADECIMIENTOS}

Se agradece a David Palacios (Pala), Pablo Solís (CONICIT) y Manuel Spinola (UNA), Rodolfo Acuña y Wendy Barrantes (ACOSA-SINAC), Emily Tour, Jademar, Quincho, Cumbia y familia Lara. A la Fundación Keto, Ministerio de Ciencia y Tecnología (MICIT) y al CONICIT, Project A.W.A.R.E, Rufford Maurice Laing Foundation, Cascadia Research Collective, U.S. Fish and Wildlife Service
(USFWS) y Cetacean Society International (CSI).

\section{RESUMEN}

De las 29 especies de cetáceos presentes en Costa Rica, se han realizado investigaciones exhaustivas solamente para tres especies. Lo anterior evidencia la poca información general existente sobre cetáceos, a pesar de que Costa Rica posee un notable crecimiento de actividades de avistamiento. Debido al uso que se está generando en las zonas costeras, cada vez se hace más necesario determinar la ocurrencia de cetáceos en zonas de alta actividad turística, con el fin de proponer medidas de conservación. En el presente estudio, se determinaron variables ambientales que estaban relacionadas con la presencia de especies registradas, a partir de 166 avistamientos entre el 2005 y 2006. Las especies con mayor proporción de avistamientos fueron Stenella attenuata (68\%), seguida por Megaptera novaeangliae $(13 \%)$ y Tursiops truncatus (10\%). La presencia del delfín manchado se relaciona con cambios de salinidad y trasparencia del agua, mientras que la de la ballena jorobada estuvo relacionada con el oleaje y la temperatura superficial del agua. Se confirma la presencia de 7 especies de cetáceos en dos zonas costeras del Pacífico de Costa Rica, de las cuales tres están presentes a lo largo del año. Se encontraron variables ambientales relacionadas con la presencia de al menos dos especies.

Palabras clave: ballenas, delfines, Costa Rica, Stenella, Tursiops, Megaptera, cetáceos, costa Pacífica.

\section{REFERENCIAS}

Acevedo-Gutiérrez, A. \& S.C. Stienessen. 2004. Bottlenose dolphins (Tursiops truncatus) increase number of whistles when feeding. Aquat. Mamm. 30: 357-362.

Acevedo-Gutiérrez, A. \& S. Burkhart. 1998. Seasonal distribution of bottlenose (Tursiops truncatus) and pantropical spotted (Stenella attenuata) dolphins (Cetacea: Delphinidae) in Golfo Dulce, Costa Rica. Rev. Biol. Trop. 46: 91-101.

Acevedo-Gutiérrez, A. \& N. Parker. 2000. Surface behavior of bottlenose dolphins is related to spatial arrangement of prey. Mar. Mam. Sci. 16: 287-298.

Acevedo-Gutiérrez, A. 1999. Aerial behavior is not a social facilitator in bottlenose dolphins hunting in small groups. J. Mammal. 80: 768-776.

Acevedo-Gutiérrez, A., B. Brennan, P. Rodríguez \& P. Thomas. 1997. Re-sightings and behavior of false killer whales (Pseudorca crassidens) in Costa Rica. Mar. Mam. Sci. 13: 307-314. 
Acevedo-Gutiérrez, A. \& M.A. Smultea. 1995. First records of humpback whales including calves at Golfo Dulce and Isla del Coco, Costa Rica, suggesting geographical overlap of Northern and Southern hemisphere populations. Mar. Mam. Sci. 11: 554-560.

Akaike, H. 1974. A new look at the statistical model identification. T. Automat. Contrl. 19: 716-723.

Brenes, C. 2001. Fundamentos de oceanografía descriptiva: aplicaciones al istmo centroamericano. Proyecto para el desarrollo integral de la pesca artesanal en la región autónoma atlántico sur. DIPAL, Nicaragua.

Brenes, C.B., L. Kwiecinski, L.D’ Croz \& J. Cháves. 1995. Características oceanográficas de la plataforma Pacífica de América Central y aguas oceánicas adyacentes. PRADEPESCA, Panamá.

Calambokidis, J., G.H. Steiger, K. Rasmussen, J.R. Urbán, K. Balcomb, P. Ladrón de Guevara, M. Salinas, J.K. Jacobsern, L.M. Herman, S. Cerchio \& J.D. Darling. 2000. Migratory destinations of humpback whales that feed off California, Oregon and Washington. Mar. Ecol. Prog. Ser. 192: 295-304.

Clapham, J. \& J. Mead. 1999. Megaptera novaeangliae. Mamm. Species 604: 1-9.

Connor, R., R. Wells, J. Mann \& A. Read. 2000. The bottlenose dolphin: social relationship in a fission-fussion society, p. 91-127. In J. Mann, R. Connor, P. Tyack \& H. Whitehead (eds.). Cetaceans Societies: field studies of dolphins and whales. The University of Chicago, Chicago, EEUU.

Cubero-Pardo, P. 2007. Distribución y condiciones ambientales asociadas al comportamiento del delfín bufeo (Tursiops truncatus) y el delfín manchado (Stenella attenuata) (Cetacea: Delphinidae) en el Golfo Dulce, Costa Rica. Rev. Biol. Trop. 55: 549-557.

Cubero-Pardo, P. 1998. Patrones de comportamiento diurnos y estacionales de Tursiops truncatus y Stenella attenuata (Mammalia: Delphinidae) en el Golfo Dulce, Costa Rica. Rev. Biol. Trop. 46: 103-110.

Escorza-Trevino S., F.I. Archer, M. Rosales, A. Lang \& A.E. Dizon. 2005. Genetic differentiation and intraspecific structure of eastern tropical Pacific spotted dolphins, Stenella attenuata, revealed by DNA analyses. Conserv. Genet. 6: 587-600.

Fertl, D., A. Acevedo-Gutierrez \& F. Darby. 1996. A report of killer whales (Orcinus orca) feeding on a carcharhinid shark in Costa Rica. Mar. Mam. Sci. 12: 606-611.
Flórez-González, L., A.J. Capella, B. Haase, G.A. Bravo, F. Félix \& T. Gerrodette. 1998. Changes in winter destinations and the Northernmost record of Southeastern Pacific humpback whales. Mar. Mam. Sci. 14: 189-196.

Garcia, C. \& S.M. Dawson. 2003. Distribution of pantropical spotted dolphins in Pacific coastal waters of Panama. LAJAM 2: 29-38.

Gerrodette, T. \& D. Palacios. 1996. Estimates of cetacean abundance in EEZ waters of the Eastern Tropical Pacific. National Marine Fisheries Service, Southwest Fisheries Science Center, La Jolla, California, EEUU.

Hoyt, E. \& M. Iñíguez. 2008. Estado del avistamiento de cetáceos en América Latina. WDCS, Chippenham, UK; IFAW, East Falmouth, EEUU; y Global Ocean, Londres, Inglaterra.

InfoStat. 2003. InfoStat versión 1.5. Manual del usuario. Grupo InfoStat, FCA, Universidad Nacional de Córdoba. Brujas, Argentina.

Janzen D.H. \& D.E. Wilson. 1983. Mammals, p. 426-442. In D.H. Janzen (ed.). Costa Rican Natural History. University of Chicago, Chicago, EEUU.

Jiménez, C. 2001. Seawater temperature measured at the surface and at two depths ( 7 and $12 \mathrm{~m}$ ) in one coral reef at Culebra Bay, Gulf of Papagayo, Costa Rica. Rev. Biol. Trop. 49: 153-161.

Martínez-Fernández, D., A. Montero-Cordero, L.J. MayCollado \& A. Calambokidis. 2005. Occurrence of Pseudorca crassidens in the Pacific coastal waters of Costa Rica, p. 181. In Abstracts 16th Biennial Conference on the Biology of Marine Mammals, San Diego. Dec. 12 - Dec. 16, 2005.

Martínez-Fernández, D. \& F. Garita-Alpízar. 2006. Presencia de orcas (Orcinus orca) en Costa Rica: Posible ocurrencia estacional en aguas del Pacífico tropical. Abstracts Primera Reunión Internacional sobre el Estudio de los Mamíferos Acuáticos SOMEMMA - SOLAMAC, 5-9 de noviembre. Mérida, México.

May-Collado, L.J. 2009. Marine Mammals, p. 479-496. In I. Wehrmann \& J. Cortes (eds). Marine Biodiversity of Costa Rica, Central America. Springer, The Netherlands.

May-Collado, L.J. \& A. Morales-Ramírez. 2005. Presencia y patrones de comportamiento del delfín manchado costero, Stenella attenuata graffmani (Cetacea: Delphinidae) en el Golfo de Papagayo, Costa Rica. Rev. Biol. Trop. 53: 265-276. 
May-Collado, L.J., T. Gerrodette, J. Calambokidis, K. Rassmusen \& I. Sereg. 2005. Distribution of cetaceans sightings in the EEZ of Costa Rica. Rev. Biol. Trop. 53: 249-263.

May-Collado, L.J. \& J. Forcada. 2001. Abundance, occurrence and behavior of the coastal spotted dolphin (Stenella attenuata graffmani) in the Northern Pacific of Costa Rica. Abstracts $14^{\text {th }}$ Biennial Conference on the Biology of Marine Mammals, Vancouver Nov. 28 - Dec. 2, 2001.

Montero-Cordero, A. \& D. Martínez-Fernández. 2007. Whale-watching revenues and decree regulation awareness in the South Pacific of Costa Rica. 17th Biennial Conference on the Biology of Marine Mammals, Cape Town. Nov. 29th- Dec. 3rd, 2007.

Montero-Cordero, A. 2007. Comportamiento del delfín manchado Stenella attenuata graffmani (Cetacea: Delphinidae) en ausencia y en presencia de botes turísticos: Evaluación biológica y socio-económica en Bahía Drake e Isla del Caño. Tesis de Maestría, Universidad de Costa Rica.

Montero-Cordero, A. \& J. Lobo. 2010. Effect of tourist vessels on the behaviour of the pantropical spotted dolphin in Drake Bay and Cano Island, Costa Rica. J. Cetacean Res. Manage.

Norris, K.S. \& T.P. Dohl. 1980. The structure and function of cetacean's schools, p. 211-261. In L.M. Herman (ed). Cetacean Behavior: Mechanism and function. John Wiley and son, Nueva York, EEUU.

Oviedo, L. 2007. Dolphin sympatric ecology in a tropical fjord: habitat partitioning by bathway and topography as a strategy to coexist. J. Mar. Biol. Ass. UK. 87: $1327-1335$

Oviedo, L. \& M. Solis. 2008. Underwater topography determines critical breeding habitat for humpback whales near Osa Peninsula, Costa Rica: implications for Marine Protected Areas. Rev. Biol. Trop. 56: 591-602.
Palacios-Alfaro, J.D. 2007. Presencia y comportamiento de dos especies de delfines en el Pacífico central de Costa Rica. Tesis de Graduación. Universidad Nacional de Costa Rica.

Perrin, W. 2002. Pantropical Spotted Dolphins, p 865867. In W. Perrin, B. Würsig \& J.G.M. Thewissen (eds.). Encyclopedia of Marine Mammals. Academic, EEUU.

Quesada-Alpízar, M. \& J. Cortés. 2006. Los ecosistemas marinos del Pacífico sur de Costa Rica: estado del conocimiento y perspectivas de manejo. Rev. Biol. Trop. 54: 101-145.

Quesada-Alpízar, M., J. Cortés, J.J. Alvarado \& A.C. Fonseca. 2006 Características hidrográficas y biológicas de la zona marino terrestre del Área de Conservación Osa. Serie Técnica: Apoyando los esfuerzos en el manejo y protección de la biodiversidad tropical. The Nature Conservancy. San Jose, Costa Rica.

R Development Core Team. 2006. R: A language and environment for statistical computing. R Foundation for Statistical Computing, Vienna, Austria. http:// www.R-project.org.

Rassmussen K., D. Palacios, J. Calambokidis, M.T. Saborio, L. Dalla Rosa, E. Secchi, R. Steiger, J. Allen \& G. Stone. 2007. Southern hemisphere whales off central america: insight from water temperature into the longest mammalian migration. Biol. Lett. 3: 302-305.

Rodríguez-Fonseca, J. \& A. Fischel-Quirós. 2007. Impacto socioeconómico del Turismo de Observación de Cetáceos en Costa Rica 2006-2007. Informe Técnico FP4-07. WSPA/Promar, San José, Costa Rica.

Steiger, G.H., J. Calambokidis, K.C. Sears \& J.C. Cubbage, 1991. Movements of humpback whales between California and Costa Rica. Mar. Mam. Sci. 7: 306-310.

Waylen, P. \& C. Caviedes. 1996. Interannual variability of monthly precipitation in Costa Rica. J. Climatel. 9: 2606-2613. 